\title{
BMJ Open Effect of e-cigarette advertisements and antismoking messages on explicit and implicit attitudes towards tobacco and e-cigarette smoking in 18-65-year-olds: a randomised controlled study protocol
}

Paula Booth, Ian P Albery, Daniel Frings

To cite: Booth P, Albery IP, Frings $D$. Effect of e-cigarette advertisements and antismoking messages on explicit and implicit attitudes towards tobacco and e-cigarette smoking in 18-65-year-olds: a randomised controlled study protocol. BMJ Open 2017;7:e014361. doi:10.1136/ bmjopen-2016-014361

- Prepublication history for this paper is available online. To view these files please visit the journal online (http://dx.doi org/10.1136/bmjopen-2016014361).

Received 19 September 2016 Revised 3 May 2017 Accepted 4 May 2017

CrossMark

Division of Psychology, School of Applied Sciences, London South Bank University, London, UK

Correspondence to

Dr. Paula Booth; p.booth@uel. ac.uk

\section{ABSTRACT}

Introduction Since the advent of e-cigarettes, e-cigarette advertising has escalated and companies are able to use marketing strategies that are not permissible for tobacco products. Research into the effect of e-cigarette advertising on attitudes towards tobacco and e-cigarettes is in its infancy. To date, no research has compared indirect (implicit) measures of attitude towards e-cigarettes with direct (explicit) measures. Furthermore, little consideration has been given to how viewing online advertisements may have an effect on attitudes towards e-cigarettes or how positive attitudes to e-cigarettes may undermine antismoking public health messages. The objectives of this study are to investigate (1) the relationship between explicit and implicit attitudes towards tobacco and e-cigarettes, (2) the effect of e-cigarette advertising on these attitudes and (3) the effect of these attitudes on the efficacy of antismoking health messages.

Methods and analysis In experiment 1 an analysis of covariance will be conducted to determine whether viewing an e-cigarette advertisement, compared with a neutral image, has an effect on implicit or explicit attitudes towards tobacco and e-cigarettes, and if these attitudes differ between smokers, vapers and non-smokers aged 18 - 25 years. In experiment 2, moderation analysis will be conducted to assess whether attitudes towards e-cigarettes moderate the psychological efficacy of antismoking health messages in participants aged 18-65 years. In each experiment, attitudes will be measured preintervention and postintervention and 1 week later $(n=150)$ in participants who are smokers $(n=50)$, vapers $(n=50)$ or non-smokers $(n=50)$.

Ethics and dissemination Approval for this study has been given by the London South Bank University's (LSBU) Research Ethics Committee. The findings of these studies will be submitted for publication and disseminated via conferences. The results will be integrated into course provision for practitioners training at LSBU.

\section{INTRODUCTION}

In the UK there are currently 2.8 million people using e-cigarettes, ${ }^{1}$ and it was estimated that in 2014 projected sales in the USA would be worth over $\$ 2$ billion. ${ }^{2}$ E-cigarettes are devices that deliver a vegetable glycerine
Strengths and limitations of this study

- This study will determine whether positive attitudes towards e-cigarettes may undermine antismoking campaign efficacy in smokers.

- This study will be one of the first to determine whether direct measures of attitudes to e-cigarettes relate to indirect measures in smokers, vapers and non-smokers.

- This study is being carried out in a carefully controlled laboratory environment and as such there is a lack of ecological validity.

and/or propylene glycerol vapour to a person when they inhale or press a button on the device. The devices are battery-operated and the vapour is supplied in a cartridge or tank which generally, but not always, contains a varying amount of nicotine. ${ }^{3}$ The act of inhalation mimics smoking a cigarette and first-generation e-cigarettes looked very much like tobacco cigarettes. Over time the appearance of e-cigarettes has evolved and becomes less like a traditional tobacco cigarette. ${ }^{4}$ Unlike regular cigarettes, e-cigarettes do not contain tobacco, thus reducing smoking-related health risks. ${ }^{5}$ However, there are concerns that e-cigarettes may be harmful to health. ${ }^{4}$ e-Cigarettes have been marketed as a smoking cessation device, ${ }^{6}$ although evidence to support this notion is inconsistent. ${ }^{2}$ Additionally, there are concerns that non-smokers may be attracted to smoking e-cigarettes and may act as a gateway to both nicotine addiction $^{7}$ and tobacco use. ${ }^{2}$

Literature suggests that attitudes towards e-cigarettes are favourable. ${ }^{8}$ Using e-cigarettes in public places is reported as more acceptable than smoking tobacco cigarettes, ${ }^{9}$ and e-cigarettes are perceived as safer, ${ }^{3810}$ healthier $^{810}$ and less addictive than tobacco cigarettes. ${ }^{10}$ 
Typically, e-cigarettes are considered a successful smoking cessation aid, ${ }^{10}$ although young adults also consider e-cigarettes to be appealing to non-smokers. ${ }^{11}$

The studies above have examined explicit, rather than implicit, attitudes towards e-cigarettes. Measures of explicit attitudes generally use direct self-report measures (eg, surveys, questionnaires or interviews) and may be influenced by social desirability biases questioning the reliability of explicit attitudes as indicators of future behaviour. ${ }^{12}$ For example, participants may be reluctant to express positive attitudes towards tobacco cigarettes because smoking tobacco cigarettes is a stigmatised behaviour and is not socially acceptable. ${ }^{12}$ However, health-related behaviours are also influenced by implicit attitudes. ${ }^{13-15}$ Implicit attitudes refer to favourable/unfavourable thoughts, feelings and behaviours towards an attitude object that are measured indirectly and reflect immediate, impulsive reactions rather than consciously controlled thought. ${ }^{16-18}$ The role of implicit and explicit attitudes in health-related behaviour is still debated as is whether the two mechanisms are mutually exclusive of one another in predicting ongoing behaviour. ${ }^{16-20}$

In general, negative explicit attitudes are expressed towards tobacco smoking by both smokers and non-smokers. ${ }^{21}$ However, for implicit measures smokers have a more positive reaction towards stimuli associated with smoking than non-smokers. ${ }^{22} 23$ Other research has found that smokers have a negative implicit attitude to smoking but that it is less negative than for non-smokers. ${ }^{2124-26}$ This suggests that smokers are more likely to show favourable attitudes towards smoking when reacting immediately and impulsively as opposed to in a more controlled conscious manner.

Various tests have been developed to capture implicit attitudes (eg, the evaluative priming task, the semantic priming task, the Go/No-Go task and the Single Category Implicit Association Test (SC-IAT) ). ${ }^{27}$ The SC-IAT is an established method of measuring implicit associations between a single target (eg, a cigarette) and two opposing attributes (bad and good). ${ }^{28}$ The implicit attitude is assessed by measuring the speed with which participants categorise a target into one of the attribute categories. The test assumes that participants will react more quickly if they automatically associate the target category with the attribute category. For example, it would be assumed that a non-smoker would react more quickly if a cigarette word was paired with a bad word, such as 'horrible', than if it were paired with a good word, such as 'love'.

This study will use SC-IATs with the personal attribute categories (SC-IAT-P) 'I like' and 'I dislike' rather than impersonal attributes, such as 'good' and 'bad', because evidence suggests that participants may be influenced by societal norms when the categories are impersonal. ${ }^{23}$ For instance, Bardin $e t a l^{24}$ found that there was no significant difference between smokers and non-smokers when labelling the attribute categories as 'pleasant' and 'unpleasant; both smokers and non-smokers had negative implicit attitudes towards cigarettes. However, when labelling the attribute category as 'I like' and I dislike', smokers' scores were neutral in comparison with the negative scores for non-smokers, and there was a significant difference between them. The design of the SC-IAT-Ps will be adapted from that used in Bardin $e t a l .^{24}$

\section{Objective 1: relationships between attitudes}

Since little research has investigated implicit attitudes towards e-cigarettes, the first objective will examine the relationship (or dissociation) between e-cigarette-related implicit and explicit attitudes. Furthermore, it has yet to be determined whether implicit or explicit attitudes towards e-cigarettes may be associated with attitudes towards tobacco cigarettes. Positive attitudes towards e-cigarettes may be related to either enhanced, depressed or neutral attitudes towards tobacco cigarettes, and these effects may be dependent on whether the attitudes towards e-cigarettes are experienced in a consciously controlled way, impulsively or both. Additionally, any association may be moderated by smoking group (smokers/non-smokers/ vapers).

\section{Objective 2: effects of e-cigarette advertisements}

The second objective tests whether viewing online e-cigarette advertising affects implicit and explicit attitudes towards e-cigarettes and tobacco cigarettes in young people.

Consistently, research has shown that viewing e-cigarette advertisements is likely to increase positive attitudes and intentions to try e-cigarettes. In a group of adolescents (13-17 years) who had never tried e-cigarettes, but who were either tobacco smokers or non-smokers, those who answered a survey after viewing an e-cigarette advert had a more positive attitude towards e-cigarettes, scored more highly on the potential benefits of e-cigarettes and had a significantly higher intention of trying e-cigarettes than those who answered the survey before being exposed to an e-cigarette advert. Additionally, current smoking of tobacco cigarettes increased the likelihood of using e-cigarettes in the future. ${ }^{29}$ Other studies comprising adult smokers ${ }^{30}$ and non-smokers ${ }^{31}$ report increased intention or more openness for trying e-cigarettes after viewing an advertisement. Conversely, Nagelhout $e t a l^{2}$ study found there was no correlation between noticing e-cigarette advertisements and e-cigarette use. However, in a study in which participants were asked to indicate if they wished to try an e-cigarette or snus (smokeless tobacco product) after viewing magazine adverts for either e-cigarettes or snus, only $16 \%$ of participants indicated that they intended to try the product. ${ }^{33}$ When they were offered a voucher for a free product at the end of the study, $45 \%$ asked for a voucher. This discrepancy may arise because participants did not want to self-report a wish to try the product and that the voucher request may be a more reliable measure of intention to try. Alternatively, participants may have had the intention of getting the free product to give to a friend/family member. It is predicted that viewing an 
e-cigarette advertisement will increase positive attitudes towards e-cigarettes in smokers, vapers and non-smokers both at an implicit and explicit level.

Findings for the effects of e-cigarette advertisements on attitudes towards tobacco smoking are less consistent. In one study, results showed that viewing an e-cigarette advertisement may encourage tobacco smokers to smoke more. Significantly more daily smokers had a tobacco cigarette while viewing an e-cigarette advertisement if it showed e-cigarette imagery, than if the auditory track of the advertisement remained but the imagery was replaced with words rather than pictures. ${ }^{34}$ Conversely, Nagelhout $e t$ al showed that noticing e-cigarette advertisements, in tobacco cigarette smokers, was associated with greater disapproval of tobacco smoking. However, there was no association between e-cigarette advertisements and tobacco cigarette quit attempts/successes. ${ }^{32}$ The current study will examine the effect of viewing an e-cigarette advertisement on attitudes towards tobacco smoking in young people. Younger people are the focus of this experiment as studies suggest that young people are deliberately targeted by e-cigarette advertisements and that this cohort is not generally using e-cigarettes as a smoking cessation tool. ${ }^{35}$ It will determine whether viewing an e-cigarette advertisement increases positive attitudes towards tobacco smoking in smokers, vapers and non-smokers, and whether these effects are found for explicit and implicit beliefs.

\section{Objective 3: the effect of attitudes towards e-cigarettes on the efficacy of antismoking messages}

Literature suggests that mass media campaigns are effective in encouraging quit attempts and reducing tobacco smoking in adults, ${ }^{36}$ but that the effect of antismoking campaigns on adolescents and young people is less robust. ${ }^{37}$ There is no published literature on whether attitudes towards e-cigarettes may moderate the efficacy of antismoking campaigns. There is a concern that favourable attitudes towards e-cigarettes may 'spill over' into attitudes towards tobacco cigarettes and undermine the efficacy of antismoking campaigns. Alternatively, having a positive attitude towards e-cigarettes may enhance the effectiveness of antismoking campaigns and encourage people to use e-cigarettes as a smoking cessation tool. The third objective will determine whether attitudes towards e-cigarettes moderate the effectiveness of antismoking messages in smokers. The age group for this experiment will be broadened to include 18-65-year-olds as quit smoking campaigns are more effective in adults as opposed to young people. ${ }^{3637}$

This programme of research will investigate these three objectives across two experimental studies by examining (1) the relationship between explicit and implicit attitudes towards e-cigarettes and tobacco cigarettes, (2) the effect of e-cigarette advertising on these attitudes and (3) the effect of attitudes towards e-cigarettes on the psychological efficacy of antihealth messages.
METHODS/DESIGN

\section{Design}

This study has an experimental design with measurements being taken from each participant at baseline, immediately postintervention and 1 week later (see figure 1 ). Two experiments are being undertaken: experiment 1 uses an image of e-cigarette advertisement as the intervention and a neutral image as the control; experiment 2 uses an image of antismoking advertisement as the intervention and a neutral image as the control. Each group of participants (smokers, non-smokers, e-cigarette users) is randomly allocated to either the control or intervention group in a 1:1 ratio by an Excel-based covariate adaptive randomisation program. ${ }^{38}$

The dependent variables are the scores derived for explicit and implicit attitudes. Explicit attitudes are being measured through a self-report questionnaire and have been previously used to measure attitudes towards e-cigarettes. ${ }^{39}$ Implicit attitudes are being measured using two SC-IAT-P tests.

\section{Participants}

In experiment 1 adults aged $18-25$ years are eligible. In experiment 2, the age range has been broadened to include participants aged 18- 65 years. Participants must either be current tobacco cigarette smokers (smoked at least 10 cigarettes in the last 30 days), current e-cigarette users (vaped at least 10 times in the last 30 days) or non-smokers (not smoked or vaped in the last 30 days). Participants are not eligible to take part if they are dual users (ie, smoke both tobacco and e-cigarettes), pregnant (or suspect pregnancy), breast feeding or have a serious medical condition. When potential participants enquire about taking part in the study, they are sent information listing these criteria. Participants are asked about their age and smoking status when they sign up for the study, and eligibility is rechecked at the first testing session. Advertising for students within a university is the primary recruitment method, but advertising locally for participants is also necessary to ensure the required sample size. Participation is voluntary, and to encourage study retention all volunteers are offered $£ 30$ on completion of all testing sessions. Alternatively, psychology undergraduate students are offered 12 participation credits towards a required total as part of their degree programme.

\section{Procedure}

The study is currently taking place in a London university single occupancy experimental laboratory. One employed researcher and/or protocol-trained intern is carrying out testing. Data collection began in March 2016 and is scheduled to finish in April 2017. Participants are tested individually. For experiment 1 participants must attend three testing sessions: a preintervention test session on day 1 , the intervention and postintervention testing on day 3 and a further postintervention session on day 10. Data collection for experiment 1 began first and feedback from participants 


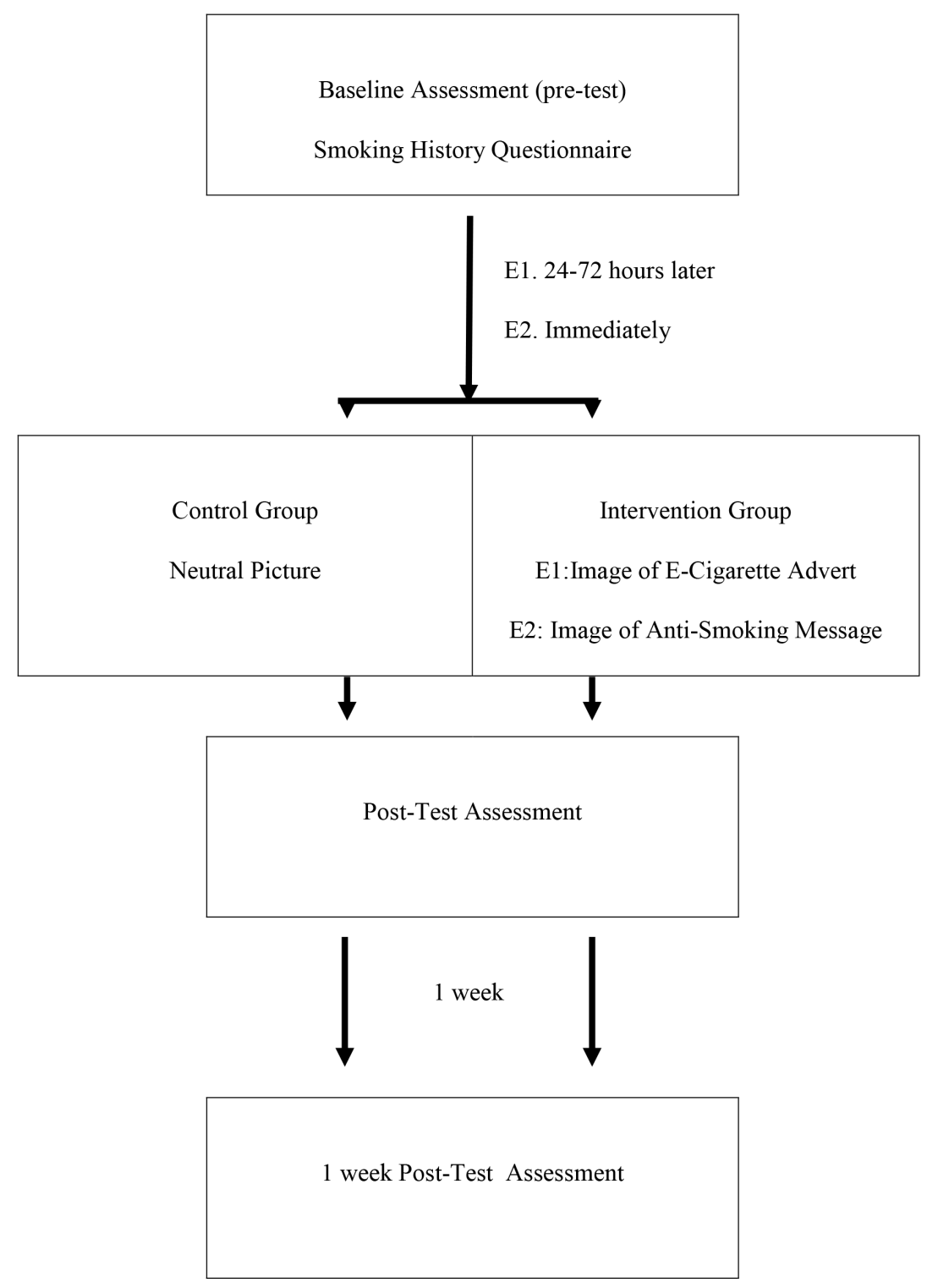

Figure 1 Study design.

suggested that recruitment might be less challenging if participants had to attend on fewer occasions. Thus, in experiment 2, participants complete both preintervention and postintervention testing on day 1 and a further postintervention session on day 7 .

On arrival at the lab participants are seated in front of a Viglen desktop computer (Viglen Genie i5 650 Processor $3.2 \mathrm{GHz}$, Viglen, Hertfordshire, UK) with an HX193DPB 19-inch LED monitor. Screening for eligibility for the study carried out by email before the testing day is then confirmed. Participants are given a copy of the study information, which has already been sent to them by email and written consent collected. First, participants are presented with the SC-IAT-P task on the computer screen. Verbal instructions are given to the participant at the beginning of the task, and understanding was checked such that the experimenter and participant are content that they are able to respond correctly. This task takes $15-20 \mathrm{~min}$. Second, the explicit attitude task is presented on the screen. These two tasks are given preintervention and postintervention and 1 week postintervention. In the preintervention session only a smoking history and beliefs questionnaire is given to the participant to complete after the explicit attitude test. After completion of the final session, participants are given a debriefing and $£ 30 /$ study credits.

\section{Intervention}

Two pilot studies have been carried out to identify an image of both (1) an e-cigarette advertisement and (2) an antismoking message for use in experiments 1 and 2. 


\section{E-cigarette advertisements}

In pilot study 1, 200 different online e-cigarette advertisement images displayed since 2013 were found. Ten themes were identified by the protocol author, depicting e-cigarettes as a smoking cessation tool, a healthy alternative, satisfying as smoking, being able to be used in places where the smoke-free legislation applies, being cool and having cosmetic appeal, being cleaner and more fragrant, being sporty, being cheaper than tobacco cigarettes, being endorsed by celebrities and improving social relationships. Each theme was given a code and every advertisement was coded by the author. Coding was undertaken independently by four research interns. Advertisements not coded consistently by at least three of the five coders were discarded. From the reduced set of 32 advertisements, the research team chose 15 advertisements based on which they found the most engaging. These images were placed in a survey developed on Qualtrics to determine which advertisement was the most persuasive and effective.

A link to the survey was placed on CrowdFlower (an online crowd-sourcing site that pays contributors to complete online research). This survey required participants to rate how persuasive, effective, happy, sad, scary, exciting and engaging they found the advertisements on a scale between 0 (not at all) and 100 (very). Additionally, control questions were built, which required participants to choose a specific rating to indicate that they were human and were paying proper attention to the survey (eg, 'are you a proper person? Please rate this answer as strongly agree'.). A total of 959 participants completed the survey. Data from participants were excluded if participants were less than 18 years old or if they did not rate the control questions. Responses from 776 participants were analysed to identify which image had the highest mean score for each adjective. One advertisement was chosen for use in experiment 1 , which had the highest score in terms of persuasiveness and effectiveness, and which was not affected by gender and did not portray cigarette smoking negatively. A control image was created by the protocol author that was very similar to the e-cigarette advertisement, but the image of the e-cigarette was replaced with an electronic toothbrush and the wording altered to advertise a toothbrush rather than an e-cigarette. The image was evaluated and agreed by the research team.

\section{Antismoking health messages}

In pilot study 2, 97 online antismoking messages were identified. Two interns identified 15 different themes: toxic gore, guns/suicide, ingredients, teen empowerment, ultimatum, lung cancer, homicide, premature ageing, amputation, teeth, recovery, children, death, expense and addiction. Each antismoking image was categorised as a theme, and after discussion between the interns one antismoking image from each theme was selected on the basis of which image they considered to be the most engaging. An identical survey to that developed in pilot study 1 was used, but the e-cigarette advertisement images were replaced with the antismoking message images.

Again, a link to the survey on Qualtrics was placed on CrowdFlower. From the original 990 participants who answered the survey, 17 were excluded for being under 18 years old, 9 because the image number was not recorded in Qualtrics and 146 because they did not rate the control questions correctly. The antismoking image with the highest scores for persuasiveness and effectiveness was chosen as the image to be used in experiment 2. The antismoking message chosen showed that physical disabilities may be caused by smoking. Three different control images were designed by the protocol author and evaluated by the research team. The chosen image showed the same image as the test intervention, but the wording was changed to state that travelling on public transport may be difficult for people with disabilities.

\section{Measures}

Experimental controls

The smoking history and beliefs survey was developed from previous literature. Current usage and dependence on tobacco cigarettes is determined by use of the Fagerström Test for Nicotine Dependence and adapted for smokers of e-cigarettes and ex-smokers. ${ }^{3}$ The strength of beliefs about e-cigarettes is measured using a 5-point Likert scale (strongly agree to strongly disagree) and has been used previously. ${ }^{37}$ Participants are asked to rate the following statements: 'using e-cigarettes can help people quit smoking', 'using e-cigarettes is less harmful to the health of the user than smoking cigarettes', and 'e-cigarettes are less addictive than cigarettes'. A scale to represent participants' motivation to quit was adapted from previous literature. ${ }^{40}$ Additionally, the number of previous quit attempts will be recorded. Awareness of e-cigarette advertising and antismoking campaigns will be measured by adapting questions originally used in a study to measure receptivity of participants to smoking advertisements. ${ }^{41}$ Participants will be asked if they recall seeing an e-cigarette advertisement recently on television, in a magazine/newspaper or on a social media site (eg, Facebook). Results will be used as covariates to control for differences in smoking history, beliefs and awareness of e-cigarette campaigns.

\section{Explicit attitudes to tobacco and e-cigarettes}

The measure is delivered via Qualtrics, an online survey tool, and completed in the lab during the testing session. Using 7-point semantic differential scales from previous work, ${ }^{19} 25$ participants are asked to indicate how bad/ good, harmful/harmless, foolish/wise, unpleasant/ pleasant, boring/exciting, not enjoyable/enjoyable, sexy/not sexy, healthy/unhealthy, glamorous/ugly and calming/stressful they think e-cigarettes/tobacco cigarettes are. The order of the required ratings will be counterbalanced. 


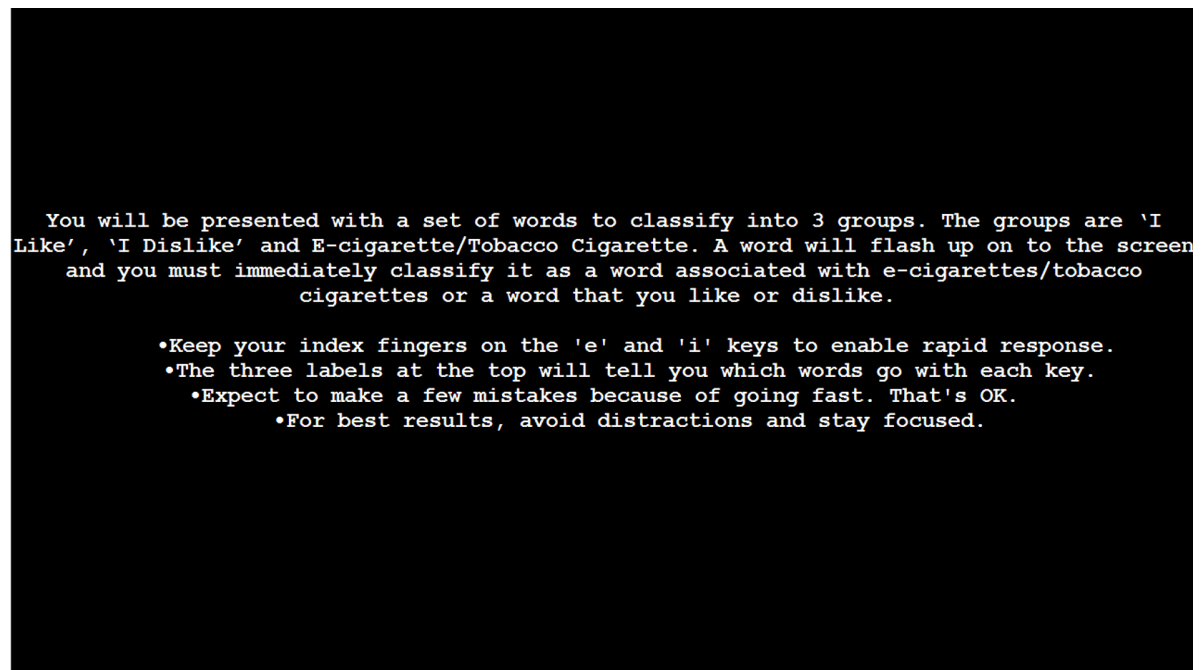

Figure 2 Screenshot of the information screen used in the Single Category Implicit Association Test with the personal attribute categories.

Implicit attitudes to tobacco and e-cigarettes

Implicit attitude is being measured using two personalised SC-IAT-P tests (for e-cigarettes and tobacco cigarettes). The labels 'I like' and 'I dislike' are presented on opposite sides at the top right-hand and left-hand side of the screen. The label 'tobacco cigarettes' or 'e-cigarettes' is presented directly under either the 'I like' or 'I dislike' label. Stimuli words, attribute category words associated with 'I like', 'I dislike' or words associated with either tobacco or e-cigarettes, appear in the middle of the screen. The participant categorises the stimuli word into one of the labels by pressing a key which represents either the left-hand side ('E' key) or the right-hand side ('O' key). Stimuli appear on the screen until participants respond or for a maximum of $1500 \mathrm{~ms} .{ }^{24}$ After this time a message 'FASTER' is presented for $500 \mathrm{~ms}$.

Seven words associated with tobacco cigarettes were drawn from previous work ${ }^{21}{ }^{42}$ : tobacco, smoking, cigarettes, smokers, ashtray, lighter and matches. A pilot study of students identified words that were most strongly associated with e-cigarettes: vapouriser, vaping, vaper, e-liquid and vape. Attribute category words were drawn from previous work ${ }^{26}$ : for the 'I dislike' category, torture, ugly, war, injury, funerals, pus, pain, infection, vomit and sad; and for the 'I like' category, sweetness, holidays, happiness, flower, happy, victory, gifts, festive, giving and beauty.
Stimuli and labels are presented in white upper-case letters ( $5 \mathrm{~mm}$ high, $4 \mathrm{~mm}$ wide) on a black background. ${ }^{28}$

Prior to each block an information screen guides participants which key will be associated with which labels (see figure 2). In each SC-IAT-P (separately for tobacco and e-cigarettes) there are four stages of four blocks (24 word trials per block); there are 24 practice trials and 72 test trials in each stage. Table 1 shows the order of label presentation. In stages 1 and 4 words are presented in a 7:7:10 ratio: tobacco/e-cigarette words plus 'I like' words versus 'I dislike' words; and in stages 2 and 3 a ratio of 7:10:7 is used. Millisecond reaction times and errors are recorded.

The algorithm used by Bardin et $a l^{24}$ and Karpinski and Steinman ${ }^{43}$ based on the D score from Greenwald et $a l^{44}$ will be used when analysing the data. Responses from practice blocks, non-responses, those less than 350 $\mathrm{ms}$ and from participants with $20 \%$ or more errors will be removed. Error responses will be replaced with the sum of the mean block time plus a $400 \mathrm{~ms}$ penalty. To determine whether participants more strongly (quickly) associate tobacco/e-cigarettes with like or dislike, the average response time for 'I like' will be subtracted from 'I dislike'. These scores will be divided by the pooled SD of all test trial times to give a standardised D score. Higher scores will indicate a more positive attitude towards

\begin{tabular}{lllll}
\hline \multicolumn{4}{l}{ Table 1} & \multicolumn{4}{l}{ Presentation of labels in the Single Category Implicit Association Test with the personal attribute categories } \\
\hline Stage & Trials & Function & 'E' key response & 'O' key response \\
\hline 1 & 24 & Practice & I like words + tobacco cigarettes & I dislike \\
& 72 & Test & I like & I dislike words + tobacco cigarettes \\
2 & 24 & Practice & & \\
& 72 & Test & I dislike words + tobacco cigarettes & I like \\
3 & 24 & Practice & Test & I like words + tobacco cigarettes \\
4 & 72 & Practice & I dislike & \\
& 24 & Test & & \\
\hline
\end{tabular}


smoking/vaping. Internal consistency of the SC-IAT-Ps will be assessed using the procedure described by Frings et $a l^{45}$

\section{STATISTICAL ANALYSIS Sample size analysis}

Based on G-Power calculation in each experiment, 150 participants will be required (50 e-cigarette users, 50 tobacco cigarette smokers and 50 non-smokers). With three time point measures and a sample of 150 spread across six groups (smoker type $\times$ intervention conditions), sufficient statistical power $(95 \%)$ will be present to detect a small effect size $(f=0.17)$ in a repeated measures interaction. An analysis of covariance (ANCOVA) between groups with six groups, no time within-subjects factor and three covariates (chosen from experimental controls measures) will be sufficiently powered $(95 \%)$ to detect a medium size effect $(f=0.32)$.

\section{Statistical analysis plan}

All data for participants who complete part 1, but not parts 2 and 3, of the study will be excluded. As recommended by Standard Protocol Items: Recommendations for Interventional Trials (SPIRIT), a full description of missing data in each condition and smoking group will be provided ${ }^{46}$ If the attrition rate, in either condition or overall, exceeds $20 \%$ for time 3 , then all time 3 data will be removed from analysis. ${ }^{47}$ If attrition is less than $20 \%$, missing data will be replaced with the 'worst ranking score' as the most conservative method. $^{48}$

\section{DATA PREPARATION}

Data will be checked to confirm that they meet the assumptions for Pearson's correlation analysis (alternative analyses will be considered if violations occur). Before analysing data using the Hayes PROCESS model, assumptions for multiple regression will be checked for multivariate normality and linearity (transformation considered if assumptions not met) and homoscedasticity. For multicollinearity between independent variables, if $r s>0.7$ one of the offending variables will be removed.

Before analysis of variance (ANOVA) data for each outcome at times 1, 2 and 3 will be assessed in terms of linearity and transformation considered if violated. If normality is violated, a non-parametric test will be used. Levene's test and Hartley's Fmax test will check homogeneity of variance. Greenhouse-Geisser statistics will be used if data violate assumptions of sphericity. Before conducting ANCOVA, covariates and explicit belief variables will be checked for multicollinearity. If any rs $>0.07$, one of the offending variables will be excluded. Analyses conducted with covariate and non-covariate models will be reported.

\section{Primary outcome measures}

Objective 1: relationships between attitudes

Correlational analyses will determine whether there is a relationship between explicit and implicit attitudes towards e-cigarettes at baseline. Additionally, the association between attitudes towards e-cigarettes and tobacco cigarettes will be investigated, and moderation analysis will test the observed relationships within each smoking group.

\section{Objective 2: effect of advertising on attitudes}

To determine whether viewing an advertisement has an effect on attitudes, mixed-model ANOVAs will be conducted with TIME (preintervention/postintervention/1 week) as a within-subjects factor and INTERVENTION (advertisement/control) and SMOKING GROUP (smokers/vapers/non-smokers) as between-subjects factor. In each experiment, one ANOVA will be conducted with implicit outcome measures as the dependent variable, and a second with the explicit test outcomes. This analysis will be repeated for attitudes towards tobacco and e-cigarettes.

Objective 3: the effect of attitudes on antismoking health message psychological efficacy

A moderation analysis using Hayes PROCESS model will determine whether attitudes towards e-cigarettes at baseline moderate the psychological efficacy of antismoking health messages on implicit/explicit attitudes towards tobacco cigarettes. Baseline tobacco scores will be covaried and the moderating effect of smoking groups will be investigated: the smoking group data will be recoded so that non-smokers' data will be compared with first vapers and second smokers.

\section{Subsidiary analysis}

Baseline current usage/dependence on tobacco/e-cigarettes, beliefs about e-cigarettes, motivation to quit, number of previous quit attempts, awareness of e-cigarette advertising and antismoking campaigns will be added as covariates. Additionally, a subsidiary analysis will be carried out to determine whether there is an effect of age on implicit and explicit attitudes towards tobacco and e-cigarettes both before and after viewing either an e-cigarette advertisement or an antismoking message.

\section{ETHICS AND DISSEMINATION}

Informed consent will be collected prior to testing. It is intended that the data set will be made available publicly via UK data repository services. Findings will be disseminated via peer-reviewed publications, scientific conferences and training events (eg, Continuing Professional Development activities). A non-technical summary will be offered to Cancer Research UK and policy/pressure organisations (eg, Action on Smoking and Health, Action on Addiction). Ethical approval has been granted from the London South Bank University Research Ethics Committee (UREC number 1602). 


\section{CONCLUSION}

This study will assess the relationship between direct and indirect measures of attitudes towards e-cigarettes and tobacco cigarettes. No known studies have previously investigated differences in implicit and explicit attitudes towards e-cigarettes. This study will show if viewing the image of an e-cigarette advertisement changes attitudes, and whether attitudes and attitude changes differ between smokers, non-smokers and e-cigarette users. Furthermore, this is the first study to consider whether positive attitudes towards e-cigarettes may potentially undermine public health campaigns encouraging smokers to quit. Findings will be important in informing policy around existing concerns about the relationship between e-cigarette attitudes and tobacco cigarettes, advertising and antismoking health campaigns. It will also inform practitioners (eg, general practitioners, cessation counsellors and so on) by providing insight into the role of e-cigarettes in smoking cessation.

Correction notice This paper has been amended since it was published Online First. Owing to a scripting error, some of the publisher names in the references were replaced with 'BMJ Publishing Group'. This only affected the full text version, not the PDF. We have since corrected these errors and the correct publishers have been inserted into the references.

Contributors DF and IPA conceptualised and designed the study and critically reviewed and revised the manuscript; PB drafted the initial manuscript and will administer the testing, data collection and statistical analysis. All authors approved the final manuscript as submitted.

Funding This work was supported by Cancer Research UK grant number C54622/ A20485.

Competing interests DF and IPA are both investigators on a randomised controlled trial funded by Allen Carr's Easyway. This trial is comparing the Allen Carr's Easway stop-smoking method with local NHS 1-1 stop smoking counselling service. The team is contractually free to publish the results regardless of the study outcome. They have no other conflicts of interest to declare.

Patient consent The study does not recruit patients.

Ethics approval London South Bank University Research Ethics Committee.

Provenance and peer review Not commissioned; externally peer reviewed.

Open Access This is an Open Access article distributed in accordance with the Creative Commons Attribution Non Commercial (CC BY-NC 4.0) license, which permits others to distribute, remix, adapt, build upon this work non-commercially, and license their derivative works on different terms, provided the original work is properly cited and the use is non-commercial. See: http://creativecommons.org/ licenses/by-nc/4.0/

(c) Article author(s) (or their employer(s) unless otherwise stated in the text of the article) 2017. All rights reserved. No commercial use is permitted unless otherwise expressly granted.

\section{REFERENCES}

1. ASH Action on Smoking and Health. Use of electronic cigarettes (vapourisers) among adults in great Britain. ASH Fact Sheet 2016;2016.

2. Schraufnagel DE, Blasi F, Drummond MB, et al. Electronic cigarettes. A position statement of the forum of international respiratory societies. Am J Respir Crit Care Med 2014;190:611-8.

3. Dawkins L, Turner J, Roberts A, et al. 'Vaping' profiles and preferences: an online survey of electronic cigarette users. Addiction 2013;108:1115-25.

4. Rom O, Pecorelli A, Valacchi G, et al. Are E-cigarettes a safe and good alternative to cigarette smoking? Ann N Y Acad Sci 2015;1340:65-74.
5. McNeill A, Brose LS, Calder R, et al. E-cigarettes: an evidence Update. A Report Commissioned by Public Health England, 2015.

6. :-.Zhu SH, Sun JY, Bonnevie E, et al. Four hundred and sixty brands of e-cigarettes and counting: implications for product regulation. Tob Control 2014;23 Suppl 3:-.iii3-iii9.

7. Durbin RJ, Waxman HA, Harkin T, et al. Gateway to addiction? A survey of popular electronic cigarette manufacturers and targeted marketing to youth. InUS Congr 2014;711.

8. Pepper JK, Brewer NT. Electronic nicotine delivery system (electronic cigarette) awareness, use, reactions and beliefs: a systematic review. Tob Control 2014;23:375-84.

9. Trumbo CW, Harper R. Orientation of US Young adults toward E-cigarettes and their use in Public. Health Behav Policy Rev 2015;2:163-70.

10. Choi K, Forster J. Characteristics associated with awareness, perceptions, and use of electronic nicotine delivery systems among young US midwestern adults. Am J Public Health 2013;103:556-61.

11. Choi K, Fabian L, Mottey N, et al. Young adults' favorable perceptions of snus, dissolvable tobacco products, and electronic cigarettes: findings from a focus group study. Am J Public Health 2012;102:2088-93.

12. Sherman SJ, Chassin L, Presson C, et al. The Intergenerational Transmission of Implicit and Explicit attitudes toward smoking. J Exp Soc Psychol 2009;45:313.

13. Sheeran P, Gollwitzer PM, Bargh JA. Nonconscious processes and health. Health Psychol 2013;32:460-73.

14. Gawronski B, Bodenhausen GV. Associative and propositional processes in evaluation: an integrative review of implicit and explicit attitude change. Psychol Bull 2006;132:692-731.

15. Wiers RW, Houben KMP. I, Roefs A, et al. 'Implicit cognition in health psychology: why common sense goes out of the window'. In: Gawronsk B, PBK, eds. Handbook of implicit social cognition. New York, NY, US: The Guilford Press, 2010:463-88.

16. Rooke SE, Hine DW, Thorsteinsson EB. Implicit cognition and substance use: a meta-analysis. Addict Behav 2008;33:131415p1328.

17. Roefs A, Huijding J, Smulders FTY, et al. Implicit measures of association in psychopathology research. Psychol Bull;137:149-93.

18. Frings D, Albery IP. The Social Identity Model of Cessation Maintenance: formulation and initial evidence. Addict Behav 2015:44:35-42.

19. Perugini M. Predictive models of implicit and explicit attitudes. $\mathrm{Br} \mathrm{J}$ Soc Psychol 2005;44:29-45.

20. Reich RR, Goldman MS, Noll JA. Using the False memory paradigm to test two Key elements of Alcohol Expectancy Theory. Exp Clin Psychopharmacol;12:102-10.

21. Huijding J, de Jong PJ, Wiers RW, et al. Implicit and explicit attitudes toward smoking in a smoking and a nonsmoking setting. Addict Behav 2005;30:949-61.

22. Huijding J, de Jong PJ. Automatic associations with the sensory aspects of smoking: positive in habitual smokers but negative in nonsmokers. Addict Behav 2006;31:182-6.

23. De Houwer J, Custers R, De Clercq A. Do smokers have a negative implicit attitude toward smoking? Cogn Emot 2006;20:1274-84.

24. Bardin B, Perrissol S, Py J, et al. Personalized SC-IAT: a possible way of reducing the influence of societal views on assessments of implicit attitude toward smoking. Psychol Rep 2014;115:13-25.

25. Swanson JE, Swanson E, Greenwald AG. Using the Implicit Association test to investigate attitude-behaviour consistency for stigmatised behaviour. Cogn Emot 2001;15:207-30.

26. Robinson M, Meier B. Smoking and the Implicit Association test: when the contrast category determines the theoretical conclusions. Basic Appl ... 2005.

27. Nosek BA, Hawkins CB, Frazier RS. Implicit social cognition: from measures to mechanisms. Trends Cogn Sci 2011:15:152-9.

28. Karpinski A, Steinman RB. The Single Category Implicit Association Test as a measure of Implicit Social cognition. J Pers Soc Psychol;91:16-32.

29. Farrelly MC, Duke JC, Crankshaw EC, et al. A Randomized Trial of the effect of E-cigarette TV advertisements on intentions to use E-cigarettes. Am J Prev Med 2015;49:686-93.

30. Kim AE, Lee YO, Shafer P, et al. Adult smokers' receptivity to a television advert for electronic nicotine delivery systems. Tob Control 2015;24:132-5

31. Popova L, Ling PM. Nonsmokers' responses to new warning labels on smokeless tobacco and electronic cigarettes: an experimental study. BMC Public Health 2014;14:997.

32. Nagelhout GE, Heijndijk SM, Cummings KM, et al. E-cigarette advertisements, and associations with the use of e-cigarettes and disapproval or quitting of smoking: findings from the International tobacco control (ITC) Netherlands Survey. Int J Drug Policy 2016;29. 
33. Smith DM, Bansal-Travers M, O'Connor RJ, et al. Associations between perceptions of e-cigarette advertising and interest in product trial amongst US adult smokers and non-smokers: results from an internet-based pilot survey. Tob Induc Dis 2015;13:14.

34. Maloney EK, Cappella JN. Does Vaping in E-Cigarette Advertisements Affect tobacco smoking urge, intentions, and perceptions in Daily, Intermittent, and former smokers? Health Commun 2016;31:1-10.

35. De Andrade M, Hastings G, Angus $\mathrm{K}$, et al. The marketing of electronic cigarettes in the Uk. $2013 \mathrm{https} / / /$ dspace.stir.ac.uk bitstream/1893/17889/1/deAndradeetale-cigsreport.pdf (accessed 26 Mar 2017).

36. Durkin S, Brennan E, Wakefield M. Mass media campaigns to promote smoking cessation among adults: an integrative review. Tob Control 2012;21:127-38.

37. Choi K, Forster JL. Beliefs and experimentation with electronic cigarettes: a prospective analysis among young adults. Am J Prev Med 2014;46:175-8.

38. Kang M, Park JH. Covariate Adaptive Randomization Program. 2007.

39. Smith DM, Bansal-Travers M, O'Connor RJ, et al. Associations between perceptions of e-cigarette advertising and interest in product trial amongst US adult smokers and non-smokers: results from an internet-based pilot survey. Tob Induc Dis $2015 ; 13: 14$

40. Zhou X, Nonnemaker J, Sherrill B, et al. Attempts to quit smoking and relapse: factors associated with success or failure from the ATTEMPT cohort study. Addict Behav 2009;34:365-73.
41. Gilpin EA, White MM, Messer K, et al. Receptivity to tobacco advertising and promotions among young adolescents as a predictor of established smoking in young adulthood. Am J Public Health 2007:97:1489-95.

42. Munafò M, Mogg K, Roberts S, et al. Selective processing of smoking-related cues in current smokers, ex-smokers and never-smokers on the modified Stroop task. J Psychopharmacol 2003;17:310-6

43. Karpinski A, Steinman RB. The single category implicit association test as a measure of implicit social cognition. J Pers Soc Psychol 2006;91:16-32.

44. Greenwald AG, Nosek BA, Banaji MR. Understanding and using the implicit association test: i. an improved scoring algorithm. J Pers Soc Psychol 2003;85:197-216.

45. Frings $D$, Melichar L, Albery IP. Implicit and explicit drinker identities interactively predict in-the-moment alcohol placebo consumption. Addict Behav Rep 2016;3:86-91.

46. Chan AW, Tetzlaff JM, Gøtzsche PC, et al. SPIRIT 2013 explanation and elaboration: guidance for protocols of clinical trials. $B M J$ 2013;346:e7586.

47. Whitlock EP, Polen MR, Green CA, et al. Behavioral counseling interventions in primary care to reduce risky/harmful alcohol use by adults: a summary of the evidence for the U.S. Preventive Services Task Force. Ann Intern Med 2004;140:557.

48. Berger VW. Conservative handling of missing data. Contemp Clin Trials 2012;33:460. 